\title{
Gain Narrowing in Few-Atom Systems
}

\author{
Tom Savels, ${ }^{1, *}$ Allard P. Mosk, ${ }^{2}$ and Ad Lagendijk ${ }^{1,2}$ \\ ${ }^{1}$ FOM Institute for Atomic and Molecular Physics, Kruislaan 407, 1098 SJ, Amsterdam, The Netherlands \\ ${ }^{2}$ Complex Photonic Systems, MESA ${ }^{+}$Research Institute, University of Twente, \\ Post Office Box 217, 7500 AE Enschede, The Netherlands
}

(Received 6 April 2006; published 5 March 2007)

\begin{abstract}
Using a density matrix approach, we study the simplest systems that display both gain and feedback: clusters of 2 to 5 atoms, one of which is pumped. The other atoms supply feedback through multiple scattering of light. We show that, if the atoms are in each other's near field, the system exhibits large gain narrowing and spectral mode redistribution. The observed phenomena are more pronounced if the feedback is enhanced. Our system is to our knowledge the simplest exactly solvable microscopic system which shows the approach to laser oscillation.
\end{abstract}

PACS numbers: 42.50.Gy, 32.80.- $\mathrm{t}$

In a laser, light is generated by a combination of light amplification by stimulated emission and optical feedback [1]. In order to study the basic physics of these processes, there has been an intensive search for laser operation in fundamental systems [2]. The resulting drive toward miniaturization has led to, among others, the realization of vertical-cavity semiconductor lasers [3], dye microsphere lasers [4], microring and microdisk semiconductor lasers [5], and photonic bandgap lasers [6]. As laser systems are made smaller, a purely macroscopic description becomes inadequate and microscopic considerations should be taken into account. An interesting example of lasers which require a (partially) microscopic treatment is the class of one-atom lasers [7], in which the gain medium is reduced to a fundamental level, while macroscopic mirrors provide feedback. Another, contrasting example is the class of random lasers [8], in which optical feedback is provided by scattering from microscopic particles, while the gain medium remains macroscopic. Obviously, neither the feedback mechanism nor the gain medium can be reduced to less than one atom.

In this Letter, we explore the most fundamental system displaying both gain and feedback: a single pumped atom, surrounded by one or more passive atoms providing optical feedback by scattering. The atoms are positioned in freespace in the absence of a cavity. The absence of a cavity and its modes differentiates our model from models in which atoms interact via a single field mode such as, e.g., atoms in a single-atom maser [9] or a superradiant laser [10]. Our few-atom system is described fully microscopically, without any quasiclassical or paraxial approximations. We show that this system, though very simple, shows surprisingly strong spectral gain narrowing and mode redistribution, indicating an approach to laser oscillation. We further demonstrate that the observed phenomena are more pronounced as the number of atoms increases, in correspondence with the intuitive $N \rightarrow \infty$ limit. The remarkable behavior of the presented $N$-atom system is related to the collective behavior of the passive atoms acting as a cavity and the pumped atom providing gain. However, the cooperativity presented here is drastically different from the type of collective behavior found in superradiance or superfluorescence [11]: while the former describes a stationary effect, the latter effect is transient. Furthermore, the spectral width of a superradiant pulse broadens with $N$, in sharp contrast to the spectral narrowing we present.

The "atoms" could be implemented as any type of subwavelength quantum objects, for example: trapped atoms, quantum dots [12], trapped ions [13], or dye molecules. Each atom interacts with the electromagnetic field by its transition dipole moment, which results in scattering of light. One of the atoms is continuously pumped, causing it to not only scatter, but also amplify the light. This $N$-atom system has optical feedback due to the fact that in the process of stimulated emission, a single atom scatters the stimulated photon isotropically, in contrast to the general notion that stimulated emission preserves the " $\mathrm{di}$ rection" of the photon, which is only true in a macroscopic gain medium [14].

We assume the atoms to be fixed in space, e.g., by a tight trap or a solid matrix. Each atom has three relevant energy levels: the ground state $a$, a highly excited state $b$, and the upper state of the relevant $c \rightarrow a$ transition $c$, as depicted in Fig. 1 (generalization to a four-level system is straight-

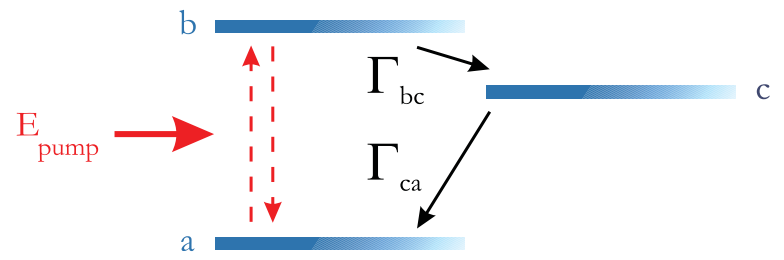

FIG. 1 (color online). The three-level system $a, b, c . \Gamma_{b c}$ and $\Gamma_{c a}$ are the decay rates from $b$ to $c$ and $c$ to $a$, respectively. Decay from $b$ to $a$ is negligible compared to other decay processes. The red dashed arrow expresses the interaction with the pump field. 
forward). One of the atoms is pumped with light which is resonant with the $b \rightarrow a$ transition. The pump intensity is expressed by the dimensionless parameter $W=$ $\Omega^{2} / \Gamma_{b c} \Gamma_{c a}$, where $\Omega=\left|\boldsymbol{d}_{b a} \cdot \boldsymbol{E}_{\text {pump }}\right| / \hbar$ is the Rabi frequency of the pump field, with $\boldsymbol{d}_{b a}$ the dipole moment of the $b \rightarrow a$ transition [15]. The decay rates from $b$ to $c$ and from $c$ to $a$ are given by $\Gamma_{b c}$ and $\Gamma_{c a}$, respectively. We can consider the atoms to be effective two-level ( $a-c)$ systems if $\Gamma_{b c} \gg \Gamma_{c a}$ and decay from $b$ to $a$ is negligible, as is usually the case in a three-level laser.

The ensemble-averaged populations of the atomic levels and the coherences between them are described by a density matrix [16] $\hat{\sigma}(t)$, whose evolution is given by the master equation

$$
\frac{d}{d t} \hat{\sigma} \equiv \hat{\mathcal{L}}_{\mathrm{nd}} \hat{\sigma}+\hat{\mathcal{L}}_{d} \hat{\sigma}
$$

with, as nondissipative operator,

$$
\hat{\mathcal{L}}_{\mathrm{nd}} \hat{\sigma} \equiv-i \sum_{i, j=1}^{N}\left[\delta^{i j} \hat{S}^{i+} \hat{S}^{j-}, \hat{\sigma}\right],
$$

and, as dissipative operator,

$$
\begin{aligned}
\hat{\mathcal{L}}_{d} \hat{\sigma} \equiv & -\frac{1}{2} \sum_{i, j=1}^{N} \Gamma^{i j}\left(\hat{\sigma} \hat{S}^{i+} \hat{S}^{j-}-\hat{S}^{i-} \hat{\sigma} \hat{S}^{j+}\right) \\
& -\frac{W}{2} \Gamma_{c a}\left(\hat{\sigma} \hat{S}^{1-} \hat{S}^{1+}-\hat{S}^{1+} \hat{\sigma} \hat{S}^{1-}\right)+\text { H.c. }
\end{aligned}
$$

The pumped atom is labeled " 1 " in expression (3). The operators $\hat{S}^{i+}$ and $\hat{S}^{i-}$, respectively, raise and lower the state of atom $i$. The atoms couple through

$$
\delta^{m n}-\frac{i}{2} \Gamma^{m n}=3 \pi \frac{\Gamma_{c a} c}{\omega_{c a}} \boldsymbol{\mu}_{m} \cdot G_{0}\left(\omega_{c a}, \boldsymbol{r}_{m}-\boldsymbol{r}_{n}\right) \cdot \boldsymbol{\mu}_{n},
$$

for the off-diagonal elements $m \neq n$. The diagonal elements of the coupling are given by $\Gamma^{n n} \equiv \Gamma_{c a}, \forall n$ and $\delta^{n n} \equiv \omega_{c a} \equiv \omega_{c}-\omega_{a}, \forall n$. The tensor $G_{0}$ represents the free-space dyadic Green function [17], $c$ is the free-space speed of light, $\boldsymbol{r}_{i}$ the position vector of atom $i$, and $\boldsymbol{\mu}_{i}$ is the normalized transition dipole moment of atom $i$. The master Eq. (1) is derived by an integration over the multimode electromagnetic field to which the atoms couple, resulting in the effective coupling term (4).

Solving the master equation (1) requires the inversion and diagonalization of the associated $2^{2 N} \times 2^{2 N}$ matrix. We solve the computer-generated symbolic master equation of the total atomic system to calculate the spectral distribution of the emitted light [18]. By applying a master equation, we ensure that both elastic and inelastic scattering of photons is taken into account in all scattering orders. Herein lies one of the major benefits of the method we use: the atomic saturation due to inelastically scattered photons is significant [19] and very difficult to incorporate in a classical scattering formalism [17].
The spectral distribution of the emitted light depends on the atoms' spatial configuration and the orientation of the transition dipole moments. We focus on configurations of atoms with interatomic distances $L$ of the order $c / \omega_{c a}$ and smaller, since for much larger distances the feedback provided by the passive atoms is limited and only a very small fraction of the photons emitted by the pumped atom will be scattered.

The average atom-photon interaction time is of the order $\Gamma_{c a}^{-1}$, while the time it takes for photons to propagate from one atom to another is of the order $\omega_{c a}^{-1} \ll \Gamma_{c a}^{-1}$. Hence, the information and energy in the system will be stored as atomic excitations rather than electromagnetic excitations. The system's storage capacity is thus determined by the number of atoms.

The spectral information of the emitted light can be deduced from the Fourier transform of the field-correlation function

$$
g^{(1)}(\tau) \equiv\left\langle: \hat{\boldsymbol{E}}^{-}(t+\tau) \cdot \hat{\boldsymbol{E}}^{+}(t):\right\rangle
$$

in steady state, where the colons denote normal and time ordering for the field operators. As a general result of the master equation, the spectrum of the emitted light can be expressed as a sum of $\frac{(2 N) !}{(N+1) !(N-1) !}$ Lorentzian contributions [20]. Each of these is characterized by a central frequency, a spectral width, and a spectral weight. The latter expresses to what extent each contribution dominates the total spectrum. If we increase the pump intensity, different modes will be subject to different gain and, consequently, modes will compete for the available population inversion in the system.

To indicate the effect of an increasing pump intensity on the spectrum of the emitted light, Fig. 2 shows the far-field spectrum for a four-atom configuration. The spectrum is averaged over a $4 \pi$ solid angle for a typical low ( $W=$ $1.77)$ and a high $(W=10.10)$ pump intensity. At low pump intensity, the emission spectrum is broad, while at higher pump intensities we observe a significant spectral narrowing.

If we wish to compare different configurations of atoms, we need to visualize the degree of observed gain narrowing. We proceed along the path originally considered by Schawlow and Townes [21] and compare the line width of the emitted light to the photon emission rate. We therefore determine the full width at half maximum $\Delta \omega$ of the farfield spectrum $I(\omega)$ averaged over a $4 \pi$ solid angle. We then evaluate the spectral weight $\int_{\Delta \omega} I(\omega) d \omega$ within the range $\Delta \omega$, which yields the total photon emission rate $n \Gamma_{c a}$ emitted in the range $\Delta \omega$. The number $n$ can then be interpreted as the number of excitations in a cavity with decay rate $\Gamma_{c a}$. Since the passive atoms can store one excitation each, the average rate $n \Gamma_{c a}$ detected in the far field cannot exceed $N \Gamma_{c a}$. Additionally, the average photon emission rate is limited by the rate $W \Gamma_{c a}\left\langle\hat{S}^{1-} \hat{S}^{1+}\right\rangle$ at which pump photons are absorbed by the system. Figure 3 is a parametric plot showing the resulting spectral width $\Delta \omega$ 


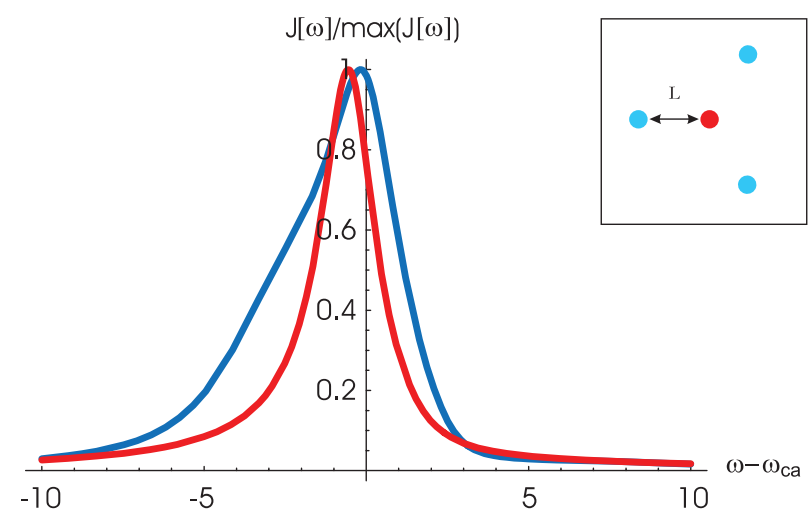

FIG. 2 (color). The normalized far-field angle-averaged spectrum versus frequency (in units of $\Gamma_{c a}$ ). The inset shows the configuration for which the spectrum is evaluated. The passive atoms (blue) are positioned in an equilateral triangle, with the pumped atom (red) in the center. All transition dipole moments are perpendicular to the plane of the atoms. The distance $L$ was chosen $0.7 c / \omega_{c a}$. The spectrum is shown for a low pump intensity $W=1.77$ (blue) and a high pump intensity $W=$ 10.10 (red).

versus $1 / n$ for the same parameters as in Fig. 2 for increasing pump intensity. We observe a large decrease of $\Delta \omega$, accompanied by an increase in $n$. Since $n$ cannot increase indefinitely, there is a critical pump intensity at which saturation of the passive atoms sets in, as shown in the inset of Fig. 3. The maximum value of $n$ is relatively low compared to $N$ due to the weak coupling between the atoms. Around the saturation point, the spectrum broadens while $n$ remains locally constant. If the pump intensity increases beyond the saturation point of the passive atoms, the emission rate decreases. This effect is due to power broadening, inherent to the three-level pumping scheme: at the saturation point of the passive atoms, an increase of the pump leads to a decoupling of the pumped atom and the passive ones, resulting in the observed decrease of $n$.

The observed relation between $1 / n$ and $\Delta \omega$ below saturation is similar to the behavior found in many macro-

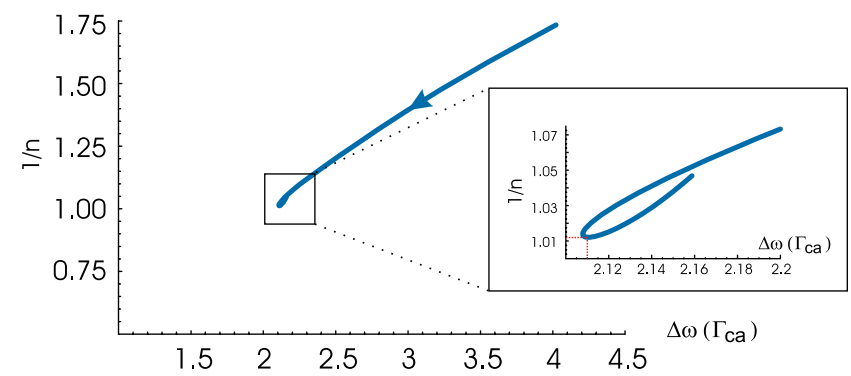

FIG. 3 (color online). The photon emission rate (relative to $\Gamma_{c a}$ ) in the range $\Delta \omega$ versus $\Delta \omega$ (in units of $\Gamma_{c a}$ ). The pump intensity $W$ ranges from 1.76 to 13.43 , while the inset focuses on the behavior around the saturation point. The arrow denotes an increase of the pump. The same configuration as in Fig. 2 is used, with $L=0.7 c / \omega_{c a}$. The red dashed lines in the inset denote the position of $\Delta \omega_{\min }$ and $n_{\max }^{-1}$. scopic lasers. As was shown by Schawlow and Townes and generalized by many others [22], the quantum-limited laser line width due to diffusion is inversely proportional to the number of photons in the laser mode. It is striking that our simple microscopic system exhibits a similar behavior while we are not in the regime typically considered in the Schawlow-Townes relation.

If the number of atoms increases, the system's storage capacity grows. Hence, the maximum value $n_{\max }$ which $n$ can attain increases with $N$. For each given number of atoms, there is an infinite number of possible configurations in which the atoms can be positioned. Since the dipole-dipole coupling depends on the configuration, both $n_{\max }$ and the corresponding width $\Delta \omega_{\min }$ will, for a given $N$, vary with the geometry.

In order to compare different configurations of atoms, we determine how many excitations can be stored with a given coherence time $\Delta \omega_{\min }^{-1}$. For each number of atoms $N$, we consider those configurations which attain their saturation point at a given value of $\Delta \omega_{\min }$. We then determine the corresponding number of excitations $n_{\max }$. Figure 4 shows the calculated $n_{\max }$ for three different values of the coherence time. For every number of atoms, different configurations exist which yield the same saturation value $\Delta \omega_{\min }$. In general, such configurations each have a different $n_{\max }$ associated with them, as represented by the identically colored symbols. We see that the effect of an increase in $N$ is twofold. First, we observe that, for a fixed coherence time, the maximum number of excitations increases with $N$. This trend indicates that, as the storage capacity of the system grows, more photons with a given coherence time $\Delta \omega_{\min }^{-1}$ can be emitted by the system. Second, when comparing different values of $\Delta \omega_{\min }$ in Fig. 4 , we see that, if the required coherence time increases, a larger capacity is

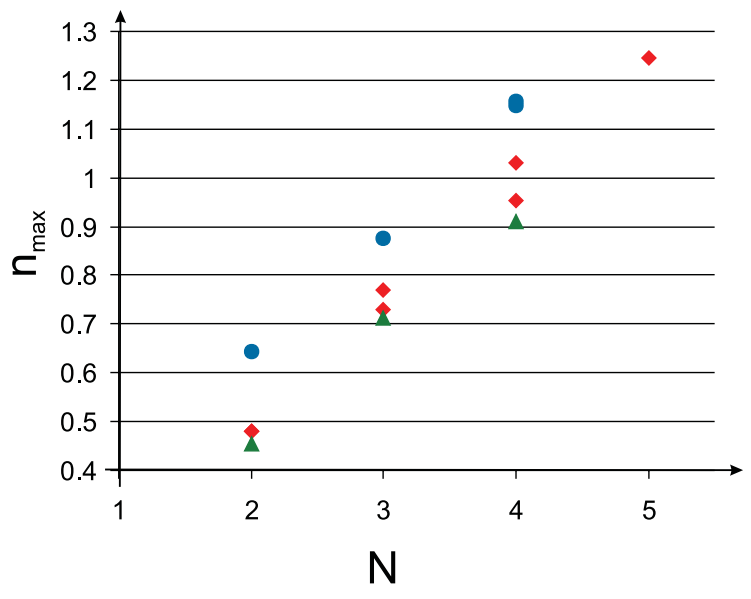

FIG. 4 (color). The relation between the maximum excitations number $n_{\max }$ and $N$. Multiple symbols for a fixed $N$ represent different configurations. Fixed colors represent fixed coherence times. The green triangles are for a coherence time $0.47 \Gamma_{c a}^{-1}$. The red diamonds are for a coherence time $0.43 \Gamma_{c a}^{-1}$. The blue circles are for a coherence time $0.29 \Gamma_{c a}^{-1}$. 
needed to attain a given number of excitations $n_{\max }$. This relation between the number of excitations and the storage capacity is in accordance with the intuitive limiting case $N \rightarrow \infty, 1 / n_{\max } \rightarrow 0$, and $\Delta \omega_{\min } \rightarrow 0$.

The efficiency with which incident pump photons are converted into photons in the range $\Delta \omega_{\min }$ is given by the ratio of the output rate $n_{\max } \Gamma_{c a}$ and the input rate $W \Gamma_{c a}\left\langle\hat{S}^{1-} \hat{S}^{1+}\right\rangle$. The numerical value of the efficiency depends on the number of atoms and the configuration, but we find as a general trend that the efficiency increases with $N$. For $\Delta \omega_{\min }^{-1}=0.43 \Gamma_{c a}^{-1}$ considered in Fig. 4, for example, the efficiency increases from $20 \%$ for $N=2$ to $24 \%$ for $N=5$. This indicates that adding more atoms leads to a better photon confinement, as we expect.

The mechanism governing light amplification in the few-atom systems presented here is stimulated emission. The spontaneous radiation emitted by the gain atom is scattered by the passive atoms; subsequent interaction with the gain atom generates stimulated emission. The presented physical processes correspond to the behavior expected of a subthreshold bad-cavity laser [22,23].

From an experimental point of view, we are convinced that our model is relevant for a wide range of realizations, such as laser cooled trapped ions [24]. Selective excitation of the ions can be achieved by tuning the polarization of the pump field and the positioning of the ions. Another possible experimental path would be to implement the atoms as quantum dots [25] which form, if bound to DNA, bioconjugated superstructures [26]. While the energy transfer in those structures is somewhat different from the one presented here, we anticipate the gain narrowing phenomenon as presented in this Letter to remain conceptually valid. As a third experimental realization, we expect our results to stimulate experimental work on cold atoms [27] interacting with pumping fields. Compared to our current system, the number of atoms in a cold-atom cloud is very high; we therefore trust the phenomena described above to be much more pronounced.

In conclusion, we described the simplest microscopic system which shows both gain and feedback. A system of only a few atoms in each other's near-field shows large gain narrowing and mode redistribution. Surprisingly, our system qualitatively exhibits behavior similar to macroscopic lasers. Adding more atoms to the system enhances the observed phenomena and allows more photons to propagate with a given coherence time.

Various extensions to our model are possible, among which schemes where more than one atom is pumped. The challenge of our method lies within the exponential scaling of the matrices involved; other approaches such as stochastical wave function calculations [28] might help overcome the current limits. Importantly, we expect our results can be tested experimentally, and we are working towards a physical realization of our model.

The authors wish to thank Gerhard Rempe and Bart van Tiggelen for fruitful discussions. This work is part of the research program of the "Stichting voor Fundamenteel Onderzoek der Materie" (FOM), which is financially supported by the "Nederlandse Organisatie voor Wetenschappelijk Onderzoek" (NWO).

*Electronic address: t.savels@amolf.nl

[1] A.E. Siegman, Lasers (University Science Books, Mill Valley, 1986); H. Haken, Laser Theory (Springer, Berlin, 1984); L. Mandel and E. Wolf, Optical Coherence and Quantum Optics (Cambridge University Press, Cambridge, England, 1995).

[2] W. E. Lamb et al., Rev. Mod. Phys. 71, S263 (1999).

[3] C. J. Chang-Hasnain, Opt. Photonics News 9, 35 (1998).

[4] H.-M. Tzeng et al., Opt. Lett. 9, 499 (1984).

[5] U. Mohideen et al., Appl. Phys. Lett. 64, 1911 (1994).

[6] R. K. Lee et al., Electron. Lett. 35, 569 (1999).

[7] K. An et al., Phys. Rev. Lett. 73, 3375 (1994); J. McKeever et al., Nature (London) 425, 268 (2003).

[8] H. Cao et al., Phys. Rev. Lett. 86, 4524 (2001); V.S. Letokhov, Sov. Phys. JETP 26, 835 (1968); N.M. Lawandy et al., Nature (London) 368, 436 (1994).

[9] D. Meschede, H. Walther, and G. Müller, Phys. Rev. Lett. 54, 551 (1985).

[10] F. Haake et al., Phys. Rev. Lett. 71, 995 (1993).

[11] M. Gross and S. Haroche, Phys. Rep. 93, 301 (1982).

[12] D. Gammon and D. G. Steel, Phys. Today 36, No. 10, 55 (2002).

[13] D. Leibfried et al., Rev. Mod. Phys. 75, 281 (2003).

[14] M.O. Scully and M.S. Zubairy, Quantum Optics (Cambridge University Press, Cambridge, England, 1997), Chap. 10.

[15] T. Savels, A. P. Mosk, and A. Lagendijk, Phys. Rev. A 71, 043814 (2005).

[16] C. Cohen-Tannoudji, J. Dupont-Roc, and G. Grynberg, Atom-Photon Interactions (Wiley, New York, 1998); R. H. Lehmberg, Phys. Rev. A 2, 883 (1970).

[17] A. Lagendijk and B. A. van Tiggelen, Phys. Rep. 270, 143 (1996).

[18] This tedious calculation involves symbolically evaluating Eq. (1) and subsequent numerical inversion and diagonalization of the generated matrix for each configuration.

[19] P. Zoller, Phys. Rev. A 20, 2420 (1979).

[20] H. Steudel, J. Phys. B 12, 3309 (1979).

[21] A.L. Schawlow and C. H. Townes, Phys. Rev. 112, 1940 (1958).

[22] S. J.M. Kuppens, M.P. van Exter, and J.P. Woerdman, Phys. Rev. Lett. 72, 3815 (1994); H. M. Wiseman, Phys. Rev. A 60, 4083 (1999).

[23] A. E. Siegman, Phys. Today 51, No. 1, 90 (1998).

[24] J. Eschner et al., Nature (London) 413, 495 (2001).

[25] B.D. Gerardot et al., Phys. Rev. Lett. 95, 137403 (2005).

[26] J. Lee, A. O. Govorov, and N. A. Kotov, Nano Lett. 5, 2063 (2005); A. Fu et al., J. Am. Chem. Soc. 126, 10832 (2004).

[27] O. Sigwarth et al., Phys. Rev. Lett. 93, 143906 (2004).

[28] K. Molmer, Y. Castin, and J. Dalibard, J. Opt. Soc. Am. B 10, 524 (1993). 